\title{
A tentative approach to the molecular structure of humic acids: The spectral evidence for a derivation of humic acids from plant-borne esters. 3. Pyrolysis-mass spectrometry
}

\author{
G. Minderman
}

Research Institute for Nature Management, Arnhem, the Netherlands

Received: 29 September 1978; accepted: 1 June 1979

Key words: humic acids, pyrolysis, spectrometry, esters

\section{Summary}

The conclusions mentioned in Parts 1 and 2 (Minderman, 1979a, b), about the formation of humic acids, are not contradicted by the results obtained by pyrolysismass spectrometry. The data support the hypothesis that humic acids can be derived from simple mixed esters, formed in the plants or by micro-organisms.

\section{Introduction}

The Curie point pyrolysis technique, followed by the uptake and analysis of the products in a mass spectrometer, was used for some substances of known structure and for a few humic acids, with the object - just as in former research with EPR ${ }^{1}$ and IR ${ }^{1}$ - not only to examine the relation between these substances and the humic acids, but also the formation and construction of humic acids. Until now, only a few publications in this field have reported favourable prospects for a more elaborate examination of the nature of humic acids (Meuzelaar et al., 1977).

\section{Methods and materials}

The method is described by Meuzelaar et al. $(1973,1974)$. The origin and preparation of the substances used were given in Parts 1 and 2 of this study (Minderman, 1979a, b). A brief description is now added to the spectra as legends. By comparing visually the agreements in the total picture of the spectra, conclusions were drawn from the mass spectrogram as a unit. Because of the still rather small resolving capacity of the apparatus, peak indication was problematic.

${ }^{1} \mathrm{EPR}=$ electron paramagnetic resonance; $\mathrm{IR}=$ infrared. 

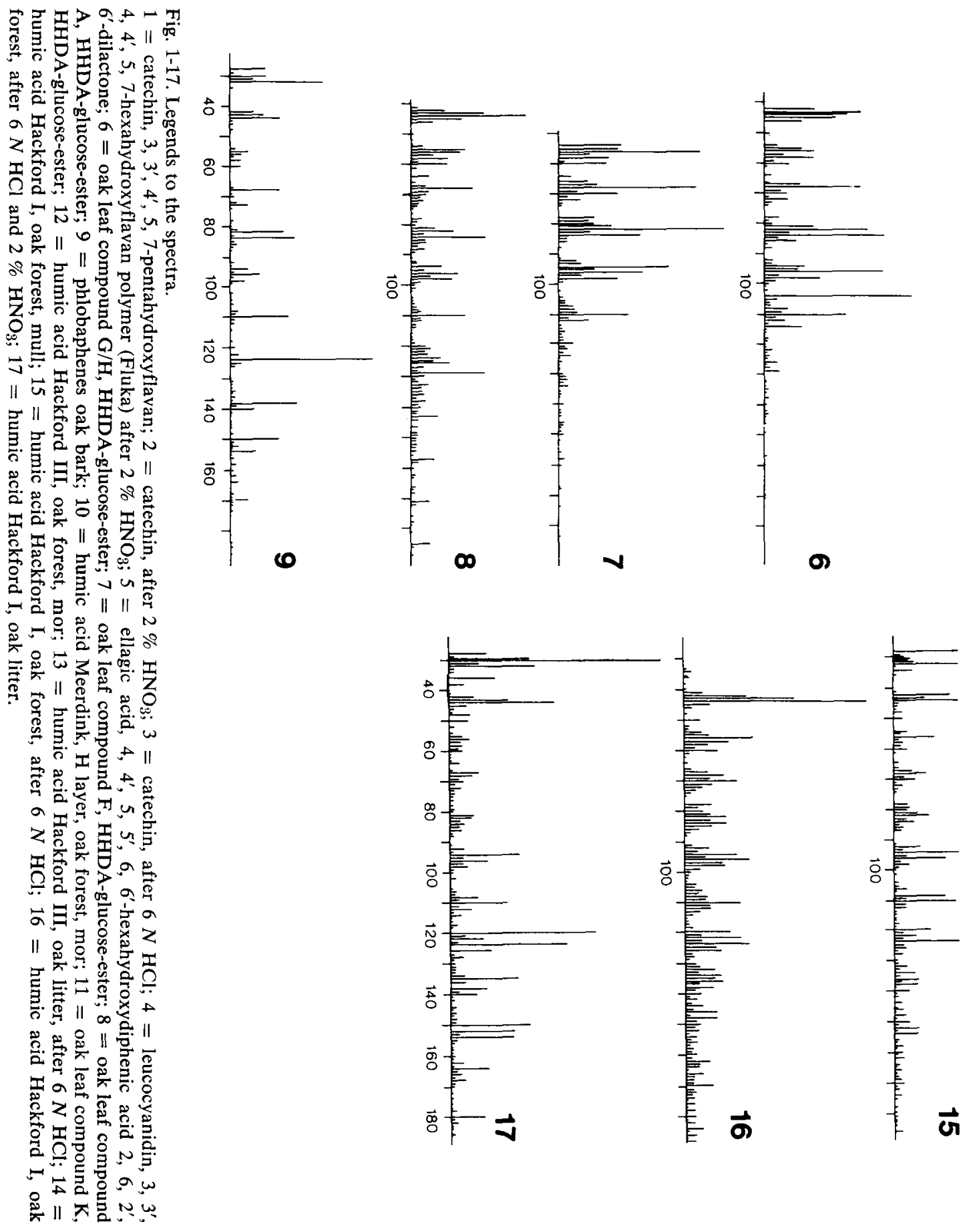
G. MINDERMAN
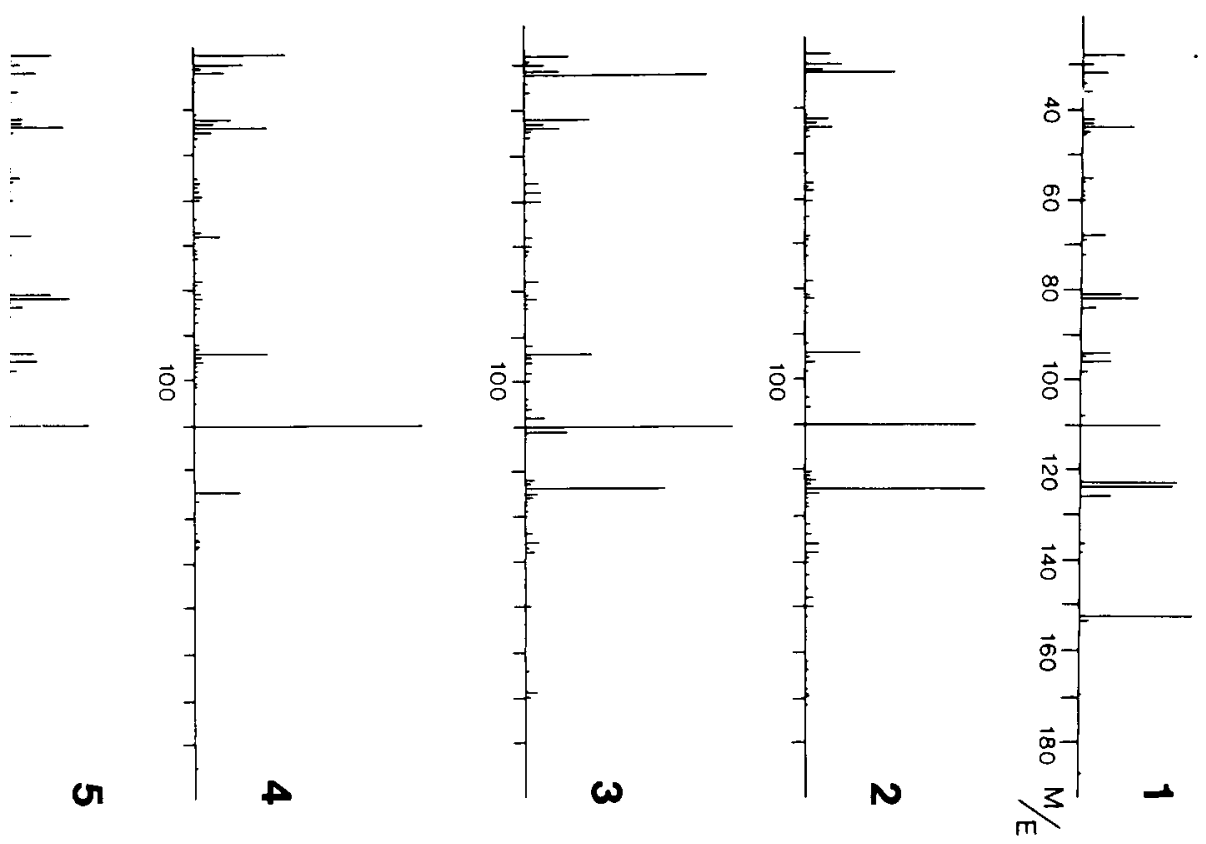

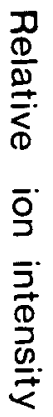

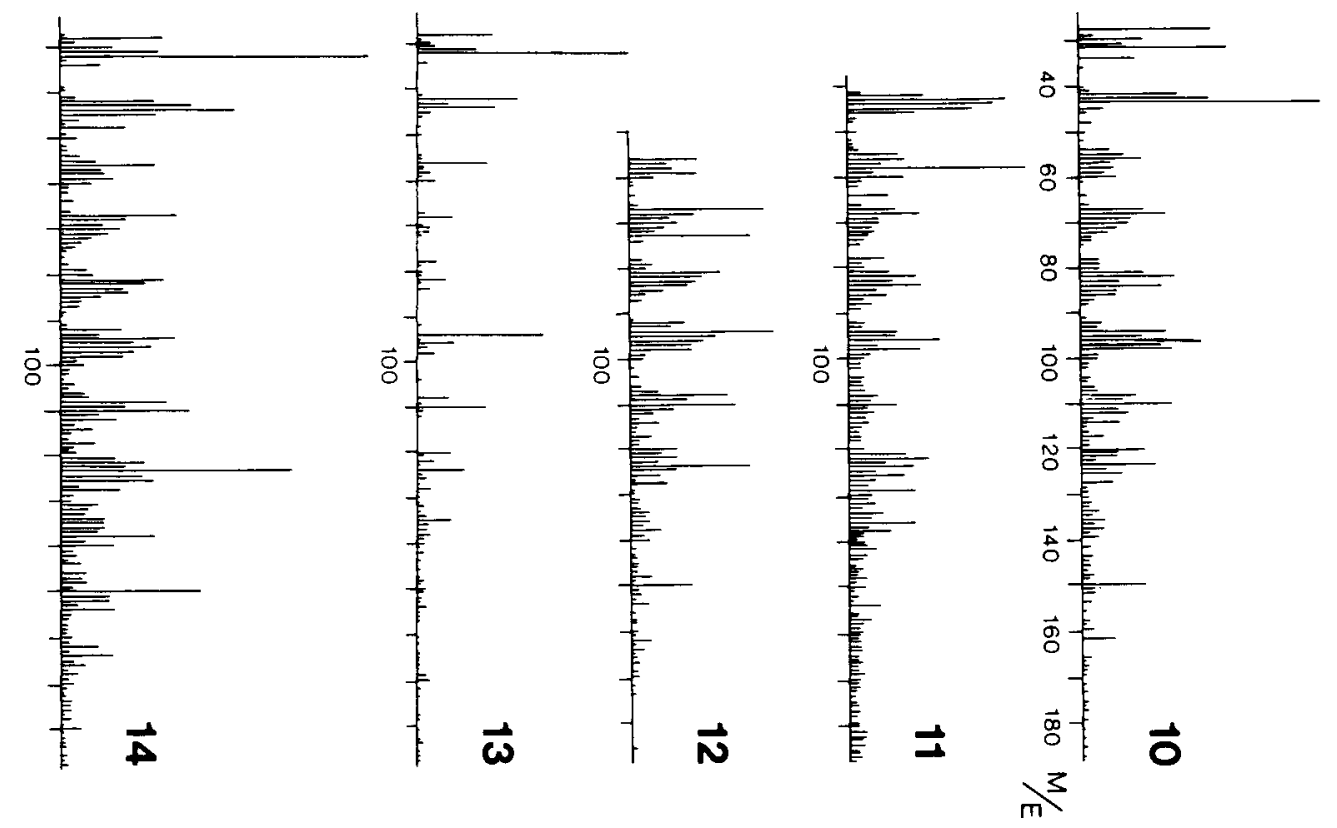

Neth. J. agric. Sci. 27 (1979)

279 


\section{Results}

It was of little use to judge whether certain mass values were present in the substances used here, or not, because nearly the same mass values could be found back in all spectra, though at different intensities. Exceptions were the masses 131 and 145, which, except for 145 in spectrum 14, were only found in the melanins and humic acids of Meuzelaar et al. (1977). In my material the additional mass 117 was found in non-hydrolysed humic acids, disappearing with hydrolysis, which indicates a hydrolysable pollution of the humic acids. Though all analyses were carried out at the Meuzelaar laboratory, it is remarkable that, in the humic acids and in the other substances now hydrolysed, mass 108 was less significant and mass 110 more significant than in material formerly examined (Haider et al., 1976; Meuzelaar et al., 1977; Nagar et al., 1975). A severe pollution of our humic acids with fulvic acid seems an unlikely explanation for the 110 peak. In the humic acid, the masses 96 and 98 were hardly present. These two are found in the spectrum of cellulose (Haider et al., 1976), but also very often in that of pure ellagic acid $\left(4,4^{\prime}, 5,5^{\prime}, 6,6^{\prime}\right.$-hexahydroxydiphenic acid dilactone) in which the other masses of the polysaccharide series also predominate. It can be seen from the spectra that the mineral acids did not affect the humic acids very much. If, for instance, humic acid 'Hackfort I' was converted into acid-boiled humic acid (ABHA) by boiling it with $6 \mathrm{~N} \mathrm{HCl}$ (spectrum 13), its spectrum could be designated as a clear phenol skeleton, the polysaccharide peaks having gone. By boiling this ABHA for one hour with $2 \% \mathrm{HNO}_{3}$, the spectrum (16) of this product again showed a kind of 'polysaccharide peak' and strongly resembled the spectrum of 'humic acid' prepared from newly fallen oak leaves. It was only the area above mass 120 that remained les pronounced. Further it could clearly be seen from the spectrum that the nitric acid has almost entirely removed the lignin. The mutual relation between the substances became clear after all spectra thought to be highly related to each other were placed beside each other. The most obvious result will then be:

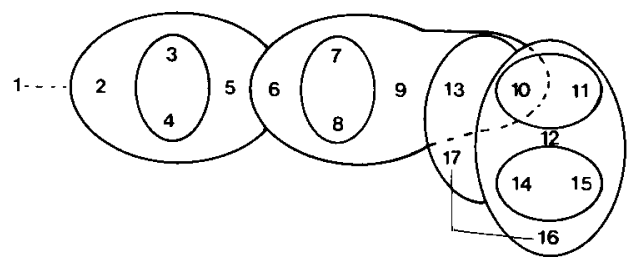

A clear series can be recognized here, to begin with catechin (1), but becoming more evident from the flavan polymers ( 2 to 4 inclusive), supplemented by ellagic acid (5) to the substances from oak leaves (6 to 8 inclusive). The series then goes from phlobaphenes (9), considered as a polycondensate of the substances 1 to 8 inclusive to the hydrolysed pre-humic acids (13) and further to the humic acids from an acid environment (10-12). The last stand closer to these substances than those coming from an alkaline environment (14). The leaf constituent $\mathrm{K}$ (11) has a spectrum strongly resembling that of humic acid from a mor $\mathrm{H}$ layer (10), while, 


\section{G. MINDERMAN}

in chemical structure, $\mathrm{K}$ is related to the other plant constituents with a mixed ester structure. Finally the spectrum of the $\mathrm{ABHA}-\mathrm{HNO}_{3}$ product (16) greatly resembles the pre-humic acids from oak leaves.

\section{Discussion}

The pyrolysis-mass spectrograms obtained showed that the substances isolated from the leaves are very similar and that they are closely related to the humic acids. Objection to these conclusions can be raised: choice and origin of all these substances were adapted to each other so that to some extent the relationship was induced. But if the spectra of the humic acids are compared with those from literature (Nagar et al., 1975; Haider et al., 1976) they have, as already observed, much in common, as in my opinion have all the spectra of the pre-humic acids. The plant substances from green oak leaves belong to the group of the ellagic tannins consisting of a sugar group in ester binding, to an aromatic dicarbonic acid; in this case, $4,4^{\prime}, 5,5^{\prime}, 6,6^{\prime}$-hexahydroxydiphenic acid. What is assumed for humic acids, namely the presence of an aromatic as well as an aliphatic part, exists in the mixed ester structure (Clark \& Tan, 1969). In Parts 1 and 2 (Minderman, 1979a, b), the above mentioned relationship was also made acceptable along other roads, namely with EPR and IR. It was found that, with EPR, the radicals present in the leaf constituents can be perceived from the green leaf via the litter, to the humic acids in the soil. Besides the relationship expressed in the IR spectra, IR also gave circumstantial evidence that, in the molecular structure of humic acids, it is above all the ester construction that prevails. The conclusion drawn from these results is, that the mixed esters in the green leaf can be considered as building stones and also as a building model of humic acids.

From literature it is certain that mixed esters of the ellagic tannin type do not occur in monocotyledonous plants, though other esters and flavonoid compounds do. With these plants, and considering humic acid formation, we must start from their breakdown products produced by microbes and fungi. From a spectral point of view, the melanins produced by moulds, strongly resemble the humic acids on the one hand (Meuzelaar et al., 1977), and the isolated mixed esters of plant substances on the other (compounds A, F, etc.). Just as with humic acids, the melanins are supposed to have a mixed ester construction. These melanins, however, must condense further although not by the action of micro-organisms as these substances are secondary metabolic products and as such cannot further be attacked by microbes. If this view is right, the micro-flora is essential for the formation of melanins in vegetations mainly consisting of monocotyledonous plants, such as meadows and steppes. For these melanins desiccation in summer may be of great importance to these pre-humic acids being condensed to humic acids by intense loss of water. This action is opposed to that occurring in a tropical rain-forest, where in a very humid environment, without irreversible desiccation, no black humic acids are formed and many substances with a low polymerizing capacity are broken down. All monomers can, in fact, mineralized by microbes; humification as such mainly involves polymerization and depolymerization of the available monomers. De- 
polymerization is hindered by the formation of $\mathrm{H}$-bridges and consequently coherence in acid environments increases. Moreover, intense desiccation is of special importance in alkaline environments as a result of which the $\mathrm{H}$-bridges can change into covalent bonds, thus greatly enhancing stability. Up to now three types of spectral analysis, reported in the three parts of this study seem to provide a basis for the hypothesis that the humic acid polymer is formed out of monomers of mixed esters. This hypothesis is supported by the following facts:

1. polyesters of sugars are known to exist (Haworth et al., 1946) and can possibly be formed in nature;

2. esters of aromatic acids show an anti-oxidant action and also work as a preservative, in other words, anti-microbially (Forgo, 1969);

3. a number of mixed esters are stable radicals (see Minderman, 1979a);

4. esters form an essential part of the final products of the secondary metabolism in plants (Harborne, 1964).

Finally, the idea is put forward that a simple building principle based on one single type of bond gives a polymer the best chance of not being attacked by microbes because such a principle will more easily escape enzymatic breakdown in nature than a multitude of principles. If humic acid had a heterogeneous construction with different types of building principles, the weakest of the bonds would most likely still be broken down enzymatically, so that the molecule would start to disintegrate. So, for the long-living humic acids in the soil a single building type here thought to be of the mixed ester type is most probable.

\section{Acknowledgments}

I wish to thank:

- Dr H. L. C. Meuzelaar of FOM Institute, Amsterdam, for his kindness in acquainting me with his new technique of analysis and for all the work he and his collaborators did in producing the spectra of all the substances I gave him;

- Jo Bierling for her scientific and technical help during the many long years of humus research;

- Roel van Beek and Julius Vermeulen for their excellent drawing and photography;

- The ladies of the typewriting department for their patience and their endurance.

\section{References}

Clark, F. E. \& K. H. Tan, 1969. Identification of a polysaccharide ester linkage in humic acid. Soil Biol. Biochem. 1: 75-82.

Forgo, I., 1969. Physikalisch-chemische Eigenschaften und Antioxydans-Wirkung einiger neuer Gallussäure-Ester-Derivate. Thesis, ETH-Zürich, No 4410. $101 \mathrm{pp}$.

Haider, K., B. R. Nagar, C. Saiz, H. L. C. Meuzelaar \& J. P. Martin, 1976. Studies on soil humic compounds, fungal melanins and model polymers by pyrolysis mass spectrometry. Proceedings FAO Conference, Braunschweig.

Harborne, J. B. (Ed.), 1964. Biochemistry of phenolic compounds. Academic Press. 618 pp. Haworth, W. N., H. Gregory \& L. F. Wiggins, 1946. Some derivatives of simple carbohydrates containing unsaturated substituents. J. chem. Soc.: 488-491. 


\section{G. MINDERMAN}

Meuzelaar, H. L. C., M. A. Posthumus, P. G. Kistemaker \& J. Kistemaker, 1973. Curie point pyrolysis in direct combination with low-Voltage electron impact ionization mass spectrometry. Analyt. Chem. 45: 1546-1549.

Meuzelaar, H. L. C., P. G. Kistemaker \& M. A. Posthumus, 1974. Recent advances in pyrolysis mass spectrometry of complex biological materials. Biomed. Mass Spectrometry 1: 312-319.

Meuzelaar, H. L. C., K. Haider, B. R. Nagar \& J. P. Martin, 1977. Comparative studies of pyrolysis mass spectra of melanins, model phenolic polymers and humic acids. Geoderma 17: 239-252.

Minderman, G., 1979a. A tentative approach to the molecular structure of humic acids: The spectral evidence for a derivation of humic acids from plant-borne esters. 1. Electron paramagnetic resonance (EPR) spectra. Neth. J. agric. Sci. 27: 79-91.

Minderman, G., 1979b. A tentative approach to the molecular structure of humic acids: The spectral evidence for a derivation of humic acids from plant-borne esters. 2. Infrared and chemical analyses. Neth. J. agric. Sci. 27: 153-175.

Nagar, B. R., E. S. Waight, H. L. C. Meuzelaar \& P. G. Kistemaker, 1975. Studies on the structure and origin of soil humic acids by Curie point pyrolysis in direct combination with low-voltage mass spectrometry. Pl. Soil 43: 681-685. 